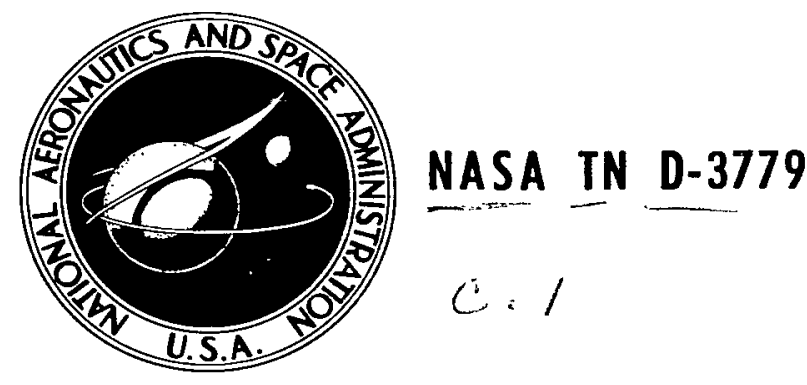

LONN COPY: RETURY TO AFW! (1?:L-S)

KIRTLAND AFB, NRIOX

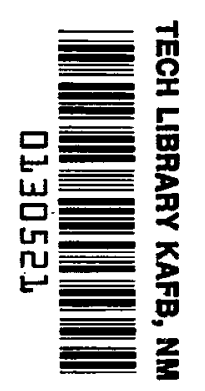

\title{
WEAK LOCALLY HOMOGENEOUS TURBULENCE AND HEAT TRANSFER WITH UNIFORM NORMAL STRAIN
}

by Robert G. Deissler

Lewis Research Center

Cleveland, Obio

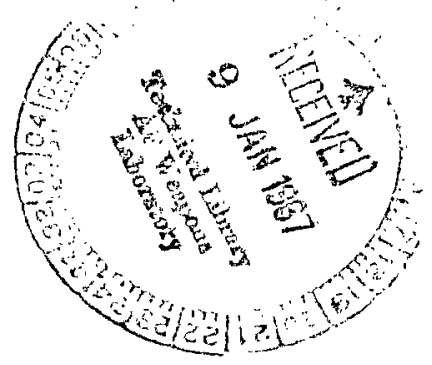

NATIONAL AERONAUTICS AND SPACE ADMINISTRATION - WASHINGTON, D. C. - JANUARY 1967 
NASA TN D-3779

WEAK LOCALLY HOMOGENEOUS TURBULENCE AND HEAT

TRANSFER WITH UNIFORM NORMAL STRAIN

By Robert G. Deissler

Lewis Research Center

Cleveland, Ohio

NATIONAL AERONAUTICS AND SPACE ADMINISTRATION

For sale by the Clearinghouse for Federal Scientific and Technical Information Springfield, Virginia 22151. - Price $\$ 1.00$ 


\title{
WEAK LOCALLY HOMOGENEOUS TURBULENCE AND HEAT \\ TRANSFER WITH UNIFORM NORMAL STRAIN \\ by Robert G. Deissler
}

Lewis Research Center

\begin{abstract}
SUMMARY
Turbulence and longitudinal heat transfer for an axisymmetric accelerating or decelerating flow were analyzed. The analysis was based on generalized two-point correlation equations, which were obtained from the incompressible Navier-Stokes, continuity, and energy equations. Viscosity, thermal conductivity, and the effects of mean strain were retained in the analysis, but the turbulence was assumed to be weak enough to neglect the triple correlation terms in the equations in comparison with the other terms. The calculated longitudinal turbulent heat transfer in the decelerating case was discussed in connection with the heat transfer at a stagnation point.
\end{abstract}

\section{INTRODUCTION}

This study is a continuation of work reported in references 1 and 2. In reference 1, weak locally homogeneous turbulence in a compressible flow with a mean longitudinal strain is analyzed. Reference 2 considers the turbulence in incompressible flow through a cone. The work in those references is, in turn, closely related to that of Pearson (ref. 3) and differs from earlier work mainly in the inclusion of the effects of viscosity and finite strain rates on the turbulence.

In the present work, the effect of uniform mean velocity gradients $\mathrm{dU}_{1} / \mathrm{dx}_{1}$, $\mathrm{dU}_{2} / \mathrm{dx}_{2}$, and $\mathrm{dU}_{3} / \mathrm{dx}_{3}$ on weak locally homogeneous turbulence and on longitudinal turbulent heat transfer is considered. Longitudinal turbulent heat transfer is of importance, for instance, in considering heat transfer near a stagnation point. Locally homogeneous herein means that the intensity of the turbulence does not vary appreciably over a correlation or mixing length. Shear stresses are assumed to be absent, and the flow is considered incompressible and axisymmetric. The turbulence portion of this work (no heat transfer) has been considered by Pearson (ref. 3). Additional results for the 
turbulence are given herein, inasmuch as Pearson gave results only for the accelerating case and did not include turbulent vorticities or spectra. Instead of a steady-state locally homogeneous but longitudinally varying turbulence, as considered herein, Pearson considered a homogeneous time-varying turbulence. The two treatments, however, give the same results.

The turbulence and turbulent heat transfer are assumed to be homogeneous in the transverse direction but only locally homogeneous in the longitudinal direction. The mean axial velocity is taken as uniform over a cross section. The turbulence is initially isotropic but is allowed to become anisotropic under the distorting influence of the mean flow. The basic equations required in the analysis will be considered in the next section.

\section{BASIC EQUATIONS}

The equations for locally homogeneous turbulent heat transfer will be considered first; the equations for the turbulence itself were obtained from reference 2 . By writing the incompressible Navier-Stokes and energy equations at two points in the turbulent fluid, reference 4 (eq. (11)) shows that

$$
\begin{aligned}
\frac{\partial \overline{\tau u_{j}^{\prime}}}{\partial t}+U_{k} \frac{\partial \overline{\tau u_{j}^{\prime}}}{\partial x_{k}}+\overline{u_{k} u_{j}^{\prime}} \frac{\partial T}{\partial x_{k}}+\frac{\partial}{\partial x_{k}} \overline{\tau u_{k} u_{j}^{\prime}}+\overline{\tau u_{k}^{\prime}} \frac{\partial U_{j}^{\prime}}{\partial x_{k}^{\prime}}+U_{k}^{\prime} \frac{\partial \overline{\tau u_{j}^{\prime}}}{\partial x_{k}^{\prime}}+\frac{\partial}{\partial x_{k}^{\prime}} \overline{\tau u_{j}^{\prime} u_{k}^{\prime}} \\
=-\frac{1}{\rho} \frac{\partial \overline{\tau p^{\prime}}}{\partial x_{j}^{\prime}}+\nu \frac{\partial^{2} \overline{\tau u_{j}^{\prime}}}{\partial x_{k}^{\prime} \partial x_{k}^{\prime}}+\alpha \frac{\partial^{2} \overline{\tau u_{j}^{\prime}}}{\partial x_{k} \partial x_{k}}
\end{aligned}
$$

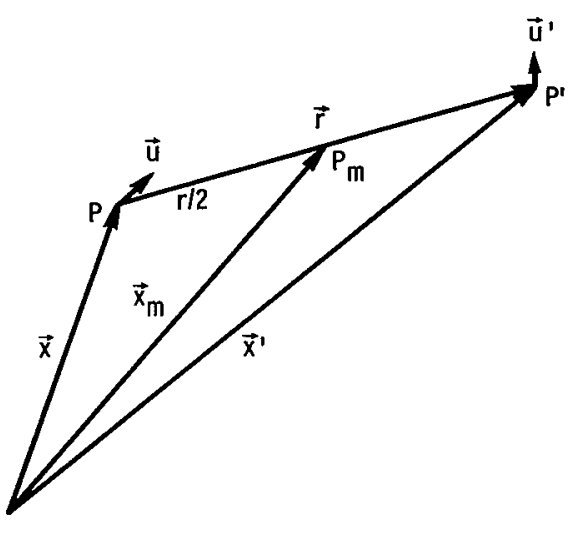

Figure 1. - Vector configuration for two-point correlation equations. where the vector configuration for correlations betweèn fluctuating quantities at points $P$ and $P^{\prime}$ is shown in figure 1. The quantity $\tau$ is the fluctuating component of the temperature at $P, u_{k}$ and $u_{j}^{\prime}$ are fluctuating velocity components at $P$ and $P^{\prime}, U_{k}$ and $U_{j}^{\prime}$ are mean velocity components, $T$ is the mean temperature at $\mathrm{P}, \mathrm{x}_{\mathrm{k}}$ and $\mathrm{x}_{\mathrm{k}}^{*}$ are space coordinates, $t$ is the time, $\rho$ is the density, $\nu$ is the kinematic viscosity, $\alpha$ is the thermal diffusivity, and $\mathrm{p}$ is the instantaneous pressure. The overbars designate correlations or averaged quantities. The subscripts can take on the values 1 , 2 , or 3 , and a repeated subscript in a term indicates a 
summation. (All symbols are defined in the appendix.) From equation (17) in reference 4,

$$
\frac{1}{\rho} \frac{\partial^{2} \overline{\tau p^{\prime}}}{\partial x_{j}^{\prime} \partial x_{j}^{\prime}}=-2 \frac{\partial U_{j}^{\prime}}{\partial x_{k}^{\prime}} \frac{\partial \overline{u_{k}^{\prime}}}{\partial x_{j}^{\prime}}-\frac{\partial^{2} \overline{\tau u_{j}^{\prime} u_{k}^{\prime}}}{\partial x_{j}^{\prime} \partial x_{k}^{\prime}}
$$

Introducing the new independent variables $r_{k} \equiv x_{k}^{\prime}-x_{k}$ and $\left(x_{k}\right)_{m} \equiv \frac{1}{2}\left(x_{k}+x_{k}^{\prime}\right)$ in equations (1) and (2) results in (fig. 1)

$$
\begin{aligned}
& \frac{\partial}{\partial t} \overline{\tau u_{j}^{\prime}}+\frac{1}{2}\left(U_{k}+U_{k}^{\prime}\right) \frac{\partial}{\partial\left(x_{k}\right)_{m}} \overline{\tau u_{j}^{\prime}}+\left(U_{k}^{i}-U_{k}\right) \frac{\partial}{\partial r_{k}} \overline{\tau u_{j}^{\prime}}+\overline{u_{k} u_{j}^{\prime}} \frac{\partial T}{\partial x_{k}}+\overline{\tau u_{k}^{\prime}} \frac{\partial U_{j}^{\prime}}{\partial x_{k}^{\prime}} \\
& +\frac{1}{2} \frac{\partial}{\partial\left(\mathrm{x}_{\mathrm{k}}\right)_{\mathrm{m}}}\left(\overline{\tau \mathrm{u}_{\mathrm{k}} \mathrm{u}_{\mathrm{j}}^{\prime}}+\overline{\left.\tau \mathrm{u}_{\mathrm{j}}^{\prime} \mathrm{u}_{\mathrm{k}}^{\prime}\right)}+\frac{\partial}{\partial \mathrm{r}_{\mathrm{k}}} \overline{\left(\tau \mathrm{u}_{\mathrm{j}}^{\prime} \mathrm{u}_{\mathrm{k}}^{\prime}\right.}-\overline{\tau \mathrm{u}_{\mathrm{k}} \mathrm{u}_{\mathrm{j}}^{\prime}}\right)=-\frac{1}{2 \rho} \frac{\overline{\partial \tau \mathrm{p}^{\prime}}}{\partial\left(\mathrm{x}_{\mathrm{j}}\right)_{\mathrm{m}}}-\frac{1}{\rho} \frac{\partial}{\partial \mathrm{r}_{\mathrm{j}}} \overline{\tau \mathrm{p}^{\prime}} \\
& +\frac{1}{4}(\nu+\alpha) \frac{\partial^{2} \overline{\tau u_{j}^{\prime}}}{\partial\left(x_{k}\right)_{m} \partial\left(x_{k}\right)_{m}}+(\nu-\alpha) \frac{\partial^{2} \overline{\tau u_{j}^{\prime}}}{\partial\left(x_{k}\right)_{m} \partial \mathbf{r}_{k}}+(\nu+\alpha) \frac{\partial^{2} \overline{\tau u_{j}^{\prime}}}{\partial \mathbf{r}_{k} \partial \mathbf{r}_{k}} \\
& \frac{1}{\rho}\left[\frac{1}{4} \frac{\partial^{2} \overline{\tau p^{\prime}}}{\partial\left(x_{j}\right)_{m} \partial\left(x_{j}\right)_{m}}+\frac{\partial^{2} \overline{\tau p^{\prime}}}{\partial\left(x_{j}\right)_{m} \partial r_{j}}+\frac{\partial^{2} \tau p^{\prime}}{\partial r_{j} \partial r_{j}}\right]=-2 \frac{\partial U_{j}^{\prime}}{\partial x_{k}^{\prime}}\left[\frac{1}{2} \frac{\partial \tau u_{k}^{p}}{\partial\left(x_{j}\right)_{m}}+\frac{\partial \tau u_{k}^{q}}{\partial r_{j}}\right] \\
& -\frac{1}{4} \frac{\partial^{2} \overline{\tau u_{j}^{\prime} u_{k}^{\prime}}}{\partial\left(x_{j}\right)_{m} \partial\left(x_{k}\right)_{m}}-\frac{1}{2} \frac{\partial^{2} \overline{\tau u_{j}^{\prime} u_{k}^{\prime}}}{\partial\left(x_{j}\right)_{m} \partial r_{k}}-\frac{1}{2} \frac{\partial^{2} \tau u_{j}^{\prime} u_{k}^{\prime}}{\partial\left(x_{k}\right)_{m} \partial r_{j}}-\frac{\partial^{2} \overline{\tau u_{j}^{\prime} u_{k}^{\prime}}}{\partial r_{j} \partial r_{k}}
\end{aligned}
$$

For locally homogeneous turbulence and turbulent heat transfer, the turbulence is considered homogeneous over a correlation length, or the scale of the inhomogeniety is much greater than the scale of the turbulence. Thus, a quantity such as $\partial^{2} \overline{\tau u_{j}^{i}} / \partial\left(x_{k}\right){ }_{m} \partial\left(x_{k}\right)_{m}$ in equation (3) will be negligible compared with $\partial^{2} \overline{\tau u_{j}^{i}} / \partial r_{k} \partial r_{k}$. (A calculation for axially decaying turbulence without mean velocity gradients, ref. 5, fig. 3 , implies that this is a good approximation except very close to the virtual origin of the turbulence.) In general, for locally homogeneous turbulence, $\partial / \partial\left(\mathbf{x}_{\mathbf{i}}\right)_{\mathbf{m}}<<\partial / \partial \mathbf{r}_{\mathbf{i}}$. Also, for that type of turbulence, the mean velocity and mean temperature will vary lin- 
early over distances for which correlations are appreciable so that $\partial \mathrm{U}_{\mathrm{j}} / \partial \mathrm{x}_{\mathrm{k}}=\partial \mathrm{U}_{\mathbf{j}} / \partial \mathrm{x}_{\mathrm{k}}=$ $\partial\left(U_{j}\right)_{m} / \partial\left(x_{k}\right)_{m}, U_{k}^{i}-U_{k}=r_{l} \partial\left(U_{k}\right)_{m} / \partial\left(x_{l}\right)_{m},\left(U_{k}+U_{k}^{i}\right) / 2=\left(U_{k}\right)_{m}$, and $\partial T / \partial x_{k}=$ $\partial \mathrm{T}_{\mathrm{m}} / \partial\left(\mathrm{x}_{\mathrm{k}}\right)_{\mathrm{m}} \cdot$ Finally, in order to make the set of equations determinate, the turbulence is assumed to be weak enough to neglect terms containing triple correlations. The turbulence in a flow with large velocity or temperature gradients may not have to be as weak as that in a flow without mean gradients. The terms containing those gradients may be large compared with triple correlation terms, even if the turbulence is moderately strong. Equations (3) and (4) become, for steady state at a fixed point,

$$
\begin{aligned}
& \overline{\tau u_{k}^{\prime}} \frac{\partial\left(U_{j}\right)_{m}}{\partial\left(x_{k}\right)_{m}}+\left(U_{k}\right)_{m} \frac{\partial}{\partial\left(x_{k}\right)_{m}} \overline{\tau u_{j}^{\prime}}+r_{l} \frac{\partial\left(U_{k}\right)_{m}}{\partial\left(x_{l}\right)_{m}} \frac{\partial}{\partial r_{k}} \overline{\tau u_{j}^{\prime}} \\
& +\overline{u_{k} u_{j}^{\prime}} \frac{\partial \mathrm{T}_{\mathrm{j}}}{\partial\left(\mathrm{x}_{\mathrm{k}}\right)_{\mathrm{m}}}=-\frac{1}{\rho} \frac{\partial}{\partial \mathrm{r}_{\mathrm{j}}} \overline{\tau \mathrm{p}^{\prime}}+(\nu+\alpha) \frac{\partial^{2} \overline{\tau \mathrm{u}_{\mathrm{j}}^{\prime}}}{\partial \mathrm{r}_{\mathrm{k}} \partial \mathrm{r}_{\mathrm{k}}} \\
& \frac{1}{\rho} \frac{\partial^{2} \overline{\tau p^{\prime}}}{\partial r_{j} \partial r_{j}}=-2 \frac{\partial\left(U_{j}\right)_{m}}{\partial\left(x_{k_{m}}\right)_{m}} \frac{\partial \overline{\tau u_{k}^{\prime}}}{\partial r_{j}}
\end{aligned}
$$

The case of uniform axisymmetric strain with no shear and with temperature gradient in the longitudinal direction is considered herein. Equation (5) for $j=1$ and equation (6) then become

$$
\begin{gathered}
\left(\mathrm{U}_{\mathrm{k}}\right)_{\mathrm{m}} \frac{\partial}{\partial\left(\mathrm{x}_{\mathrm{k}}\right)_{\mathrm{m}}} \overline{\tau \mathrm{u}_{1}^{\prime}}+\mathrm{s}_{(l)} \mathrm{r}_{l} \frac{\partial}{\partial \mathrm{r}_{l}} \overline{\tau \mathrm{u}_{1}^{\prime}}+\overline{\mathrm{bu} \mathrm{u}_{1}^{\prime} \mathrm{u}_{1}^{\prime}}+\mathrm{s}_{(1)} \overline{\tau \mathrm{u}_{1}^{\prime}}=-\frac{1}{\rho} \frac{\partial}{\partial \mathrm{r}_{1}} \overline{\tau \mathrm{p}^{\dagger}}+(\nu+\alpha) \frac{\partial^{2} \tau \mathrm{u}_{1}^{\prime}}{\partial \mathrm{r}_{\mathrm{k}} \partial \mathrm{r}_{\mathrm{k}}} \\
\frac{1}{\rho} \frac{\partial^{2} \overline{\tau \mathrm{p}^{\prime}}}{\partial \mathrm{r}_{\mathrm{j}} \partial \mathrm{r}_{\mathrm{j}}}=-2 \mathrm{~s}(l) \frac{\overline{\partial \tau \mathrm{u}_{l}^{\prime}}}{\partial \mathrm{r}_{l}}
\end{gathered}
$$

where (l) is not a tensor subscript. Equations (7) and (8) can be converted to spectral form by introducing the usual three-dimensional Fourier transforms defined as follows: 


$$
\begin{aligned}
& \overline{u_{i} u_{j}^{p}}=\int_{-\infty}^{\infty} \varphi_{i j} e^{i \vec{\kappa} \cdot \vec{r}} d \vec{\kappa} \\
& \overline{\tau u_{j}^{p}}=\int_{-\infty}^{\infty} \gamma_{j} e^{i \vec{k} \cdot \vec{r}} d \vec{\kappa} \\
& \overline{\tau p^{\prime}}=\int_{-\infty}^{\infty} \zeta^{\prime} e^{i \vec{k} \cdot \vec{r}} d \vec{\kappa}
\end{aligned}
$$

Then, using continuity and the inverse transform of equation (10) gives

$$
\mathrm{s}_{(l)} \mathrm{r}_{l} \frac{\partial}{\partial \mathbf{r}_{l}} \overline{\tau \mathbf{u}_{\mathrm{j}}^{\mathbf{j}}}=-\int_{-\infty}^{\infty} \mathrm{s}_{(l)} \kappa_{l} \frac{\partial \gamma_{\mathbf{j}}}{\partial \kappa_{l}} \mathrm{e}^{\mathrm{i} \vec{\kappa} \cdot \overrightarrow{\mathbf{r}}} \mathrm{d} \vec{\kappa}
$$

where $\vec{\kappa}$ is a wave number vector having the dimension $1 /$ length and $d \vec{\kappa}=d \kappa_{1} d \kappa_{2} d \kappa_{3}$. Taking the Fourier transforms of equations (7) and (8) results in

$$
\left(\mathrm{U}_{\mathrm{k}}\right)_{\mathrm{m}} \frac{\partial \gamma_{1}}{\partial\left(\mathrm{x}_{\mathrm{k}}\right)_{\mathrm{m}}}-\mathrm{s}_{(l)} \kappa_{l} \frac{\partial \gamma_{1}}{\partial \kappa_{l}}+\mathrm{b} \varphi_{11}+\mathrm{s}_{(1)} \gamma_{1}=2 \mathrm{~s}_{(l)} \frac{{ }^{\kappa}{ } \kappa_{l}}{\kappa^{2}} \gamma_{1}-(\alpha+\nu) \kappa^{2} \gamma_{1}
$$

where two equations have been combined into one by eliminating $\zeta^{\prime}$.

For axisymmetric strain, $\mathrm{s}_{(2)}=\mathrm{s}_{(3)}$; and by continuity of the mean flow,

$$
s_{(2)}=s_{(3)}=-\left(\frac{1}{2}\right) s_{(1)}
$$

The turbulence is also assumed to be homogeneous in the transverse direction and changes only in the longitudinal or $\mathrm{x}_{1}$ direction, so that

$$
\left(\mathrm{U}_{\mathrm{k}}\right)_{\mathrm{m}} \frac{\partial \gamma_{1}}{\partial\left(\mathrm{x}_{\mathrm{k}}\right)_{\mathrm{m}}}=\left(\mathrm{U}_{1}\right)_{\mathrm{m}} \frac{\partial \gamma_{1}}{\partial\left(\mathrm{x}_{1}\right)_{\mathrm{m}}}
$$

To simplify the notation, let $\left(\mathrm{U}_{1}\right)_{\mathrm{m}} \equiv \mathrm{U},\left(\mathrm{x}_{1}\right)_{\mathrm{m}} \equiv \mathrm{x}$, and $\mathrm{s}_{(1)} \equiv \mathrm{s}$ in the remainder of the paper. Then equation (13) becomes 


$$
\mathrm{U} \frac{\partial \gamma_{1}}{\partial \mathrm{x}}-\mathrm{s} \kappa_{1} \frac{\partial \gamma_{1}}{\partial \kappa_{1}}+\frac{1}{2} \mathrm{~s} \kappa_{2} \frac{\partial \gamma_{1}}{\partial \kappa_{2}}+\frac{1}{2} \mathrm{~s} \kappa_{3} \frac{\partial \gamma_{1}}{\partial \kappa_{3}}=-\mathrm{b} \varphi_{11}-\mathrm{s} \gamma_{1}+3 \mathrm{~s} \frac{\kappa_{1}^{2}}{\kappa^{2}} \gamma_{1}-(\alpha+\nu) \kappa^{2} \gamma_{1}
$$

where use was made of the continuity relation in the form $\kappa_{2} \gamma_{2}+\kappa_{3} \gamma_{3}=-\kappa_{1} \gamma_{1}$. Corresponding expression for $\varphi_{11}$ and $\varphi_{\mathrm{ii}}$ are given as equation (17) and (18) in reference 2. These expressions are

$$
\frac{\mathrm{U}}{\mathrm{s}} \frac{\partial \varphi_{11}}{\partial \mathrm{x}}-\kappa_{1} \frac{\partial \varphi_{11}}{\partial \kappa_{1}}+\frac{1}{2} \kappa_{2} \frac{\partial \varphi_{11}}{\partial \kappa_{2}}+\frac{1}{2} \kappa_{3} \frac{\partial \varphi_{11}}{\partial \kappa_{3}}=\varphi_{11}\left(6 \frac{\kappa_{1}^{2}}{\kappa^{2}}-2-\frac{2 \nu}{\mathrm{s}} \kappa^{2}\right)
$$

and

$$
\frac{\mathrm{U}}{\mathrm{s}} \frac{\partial \varphi_{\mathrm{ii}}}{\partial \mathrm{x}}-\kappa_{1} \frac{\partial \varphi_{\mathrm{ii}}}{\partial \kappa_{1}}+\frac{1}{2} \kappa_{2} \frac{\partial \varphi_{\mathrm{ii}}}{\partial \kappa_{2}}+\frac{1}{2} \kappa_{3} \frac{\partial \varphi_{\mathrm{ii}}}{\partial \kappa_{3}}=-3 \varphi_{11}+\varphi_{\mathrm{ii}}-2 \frac{\nu}{\mathrm{s}} \kappa^{2} \varphi_{\mathrm{ii}}
$$

For uniform $s=d U / d x$,

$$
c \equiv \frac{\mathrm{U}}{\mathrm{U}_{0}}=1+\frac{\mathrm{s}\left(\mathrm{x}-\mathrm{x}_{0}\right)}{\mathrm{U}_{0}}=1+\mathrm{x}^{*}
$$

Equations (16), (17), and (18) can be written in terms of $c$ as

$$
\begin{gathered}
\mathrm{c} \frac{\partial \gamma_{1}}{\partial \mathrm{c}}-\kappa_{1} \frac{\partial \gamma_{1}}{\partial \kappa_{1}}+\frac{1}{2} \kappa_{2} \frac{\partial \gamma_{1}}{\partial \kappa_{2}}+\frac{1}{2} \kappa_{3} \frac{\partial \gamma_{1}}{\partial \kappa_{3}}=-\frac{\mathrm{b}}{\mathrm{s}} \varphi_{11}+\gamma_{1}\left[3 \frac{\kappa_{1}^{2}}{\kappa^{2}}-1-\frac{(\alpha+\nu)}{\mathrm{s}} \kappa^{2}\right] \\
\mathrm{c} \frac{\partial \varphi_{11}}{\partial \mathrm{c}}-\kappa_{1} \frac{\partial \varphi_{11}}{\partial \kappa_{1}}+\frac{1}{2} \kappa_{2} \frac{\partial \varphi_{11}}{\partial \kappa_{2}}+\frac{1}{2} \kappa_{3} \frac{\partial \varphi_{11}}{\partial \kappa_{3}}=\varphi_{11}\left(6 \frac{\kappa_{1}^{2}}{\kappa^{2}}=2-\frac{2 \nu}{\mathrm{s}} \kappa^{2}\right) \\
\mathrm{c} \frac{\partial \varphi_{\mathrm{ii}}}{\partial \mathrm{c}}-\kappa_{1} \frac{\partial \varphi_{\mathrm{ii}}}{\partial \kappa_{1}}+\frac{1}{2} \kappa_{2} \frac{\partial \varphi_{\mathrm{ii}}}{\partial \kappa_{2}}+\frac{1}{2} \kappa_{3} \frac{\partial \varphi_{\mathrm{ii}}}{\partial \kappa_{3}}=-3 \varphi_{11}+\varphi_{\mathrm{ii}}\left(1-2 \frac{\nu}{\mathrm{s}} \kappa^{2}\right)
\end{gathered}
$$




\section{SOLUTIONS OF SPECTRAL EQUATIONS}

For solving equations (20) to (22), the turbulence is assumed to be initially isotropic (at $c=1$ ), and

$$
\left(\varphi_{i j}\right)_{0}=\frac{J_{0}}{12 \pi^{2}}\left(\kappa^{2} \delta_{i j}-\kappa_{i} \kappa_{j}\right)
$$

where $J_{0}$ is a constant that depends on initial conditions (ref. 5, eq. (43)). For the initial condition on $\gamma_{1}($ at $c=1$ ), it is assumed that

$$
\left(\gamma_{1}\right)_{0}=0
$$

That is, the turbulence-producing grid is assumed to be unheated, so that the temperature fluctuations are produced by the interaction of the mean longitudinal temperature gradient with the turbulence. Equation (23) appears to be the simplest condition that gives results that, at all values of $\mathrm{x}$, reduce to those for isotropic turbulence as the mean strain goes to zero. The use of that initial condition for $\varphi_{\mathbf{i j}}$ implies that Pearson's parameter $\nu \kappa_{0}^{2} / \mathrm{s}$ approaches $\infty$, where $\kappa_{0}$ is a characteristic initial wave number of the turbulence (ref. 3). Thus, the present results should be applicable for large kinematic viscosity, small initial turbulence scale, or small strain rate. The case $\nu \kappa_{0}^{2} / s \rightarrow \infty$ was not considered by Pearson.

Equations (20) to (22) can be solved by methods similar to those given in reference 6. Solutions of these equations subject to the initial conditions given in equations (23) and (24) are, in dimensionless form,

$$
\begin{aligned}
\gamma_{1}^{*}=-\frac{1}{12 \pi^{2}} \frac{\mathrm{c}^{-5}}{(\mathrm{c}-1)} \frac{\kappa_{2}^{*^{2}}+\kappa_{3}^{*^{2}}}{\kappa^{*^{2}}}\left(\mathrm{c}^{3} \kappa_{1}^{*^{2}}+\kappa_{2}^{*^{2}}+\kappa_{3}^{*^{2}}\right)^{2} & \\
& \times \exp \left\{-\left(\frac{1}{\mathrm{Pr}}+1\right)\left[\frac{1}{2}(\mathrm{c}+1) \kappa_{1}^{*^{2}}+\mathrm{c}^{-1}\left(\kappa_{2}^{* 2}+\kappa_{3}^{*^{2}}\right)\right]\right\} \int_{1}^{\mathrm{c}} \frac{\xi}{\kappa_{1}^{*^{2}}+\mathrm{c}^{-3} \xi^{3}\left(\kappa_{2}^{* 2}+\kappa_{3}^{* 2}\right)} \\
& \times \exp \left\{\left(\frac{1}{\mathrm{Pr}}-1\right)(\mathrm{c}-1)^{-1}\left[\frac{1}{2} \kappa_{1}^{* 2}\left(\frac{\mathrm{c}}{\xi}\right)^{2}\left(\xi^{2}-1\right)+\left(\kappa_{2}^{* 2}+\kappa_{3}^{*^{2}}\right) \mathrm{c}^{-1}(\xi-1)\right]\right\} \mathrm{d} \xi
\end{aligned}
$$


$\varphi_{11}^{*}=\frac{1}{12 \pi^{2}}\left(\kappa_{2}^{* 2}+\kappa_{3}^{* 2}\right) \frac{\mathrm{c}^{-3}}{\kappa^{*^{4}}}\left(\mathrm{c}^{3} \kappa_{1}^{* 2}+\kappa_{2}^{* 2}+\kappa_{3}^{* 2}\right)^{2} \exp \left\{-2\left[\frac{1}{2}(1+\mathrm{c}) \kappa_{1}^{* 2}+\mathrm{c}^{-1}\left(\kappa_{2}^{* 2}+\kappa_{3}^{* 2}\right)\right]\right\}$

$$
\begin{array}{r}
\varphi_{\mathrm{ii}}^{*}=\frac{1}{12 \pi^{2}}\left[\frac{\mathrm{c}^{-3}}{\kappa^{*^{2}}}\left(\mathrm{c}^{3} \kappa_{1}^{*^{2}}+\kappa_{2}^{*^{2}}+\kappa_{3}^{*^{2}}\right)^{2}+\mathrm{c}^{3} \kappa_{1}^{*^{2}}+\kappa_{2}^{*^{2}}+\kappa_{3}^{*^{2}}\right] \\
\exp \left\{-2\left[\frac{1}{2}(1+\mathrm{c}) \kappa_{1}^{*^{2}}+\mathrm{c}^{-1}\left(\kappa_{2}^{*^{2}}+\kappa_{3}^{* 2}\right)\right]\right\}
\end{array}
$$

where

$$
\begin{gathered}
\kappa_{\mathrm{i}}^{*}=\left[\frac{\nu\left(\mathrm{x}-\mathrm{x}_{0}\right)}{\mathrm{U}_{0}}\right]^{1 / 2} \kappa_{\mathrm{i}} \\
\varphi_{\mathrm{ij}}^{*}=\frac{\left(\mathrm{x}-\mathrm{x}_{0}\right) \nu}{\mathrm{J}_{0} \mathrm{U}_{0}} \varphi_{\mathrm{ij}} \\
\gamma_{1}^{*}=\frac{\nu}{\mathrm{J}_{0} \mathrm{~b}} \gamma_{1} \\
\operatorname{Pr}=\frac{\nu}{\alpha}
\end{gathered}
$$

It can be seen that $\gamma_{1}^{*}, \varphi_{11}^{*}$, and $\varphi_{\mathrm{ii}}^{*}$ are functions only of $\mathrm{c}, \kappa_{\mathbf{i}}^{*}$, and Frandtl number. For $\operatorname{Pr}=1$, equation (25) can be integrated to give

$$
\begin{aligned}
& \gamma_{1}^{*}=\frac{1}{12 \pi^{2}}(c-1)^{-1} c^{-3}\left(\frac{\kappa_{2}^{* 2}+\kappa_{3}^{* 2}}{\kappa_{1}^{* 2}}\right)^{1 / 3} \kappa^{*-2}\left(\mathrm{c}_{1}^{3} \kappa_{1}^{* 2}+\kappa_{2}^{* 2}+\kappa_{3}^{* 2}\right)^{2}
\end{aligned}
$$

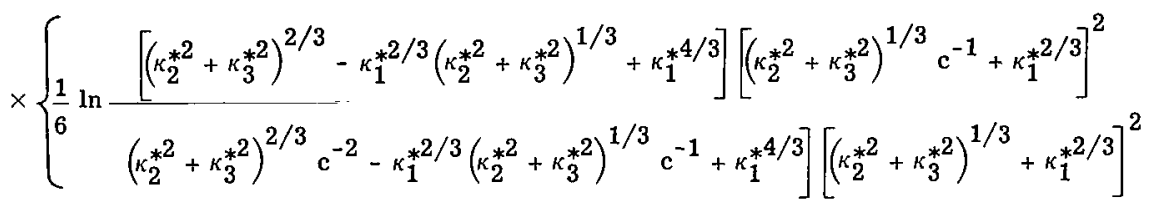

$$
\begin{aligned}
& \left.+\frac{1}{\sqrt{3}} \tan ^{-1}\left[\frac{2\left(\kappa_{2}^{* 2}+\kappa_{3}^{* 2}\right)^{1 / 3}}{\sqrt{3} \kappa_{1}^{* 2 / 3}}-\kappa_{1}^{*_{1}^{2 / 3}}\right]-\frac{1}{\sqrt{3}} \tan ^{-1}\left[\frac{2\left(\kappa_{2}^{*_{2}^{2}}+\kappa_{3}^{* 2}\right)^{1 / 3}}{\sqrt{3} \kappa_{1}^{2 / 3}} \mathrm{c}^{-1}-\kappa_{1}^{* 2 / 3}\right]\right\} \exp \left\{-2\left[\frac{1}{2}(1+\mathrm{c}) \kappa_{1}^{*^{2}}+\mathrm{c}^{-1}\left(\kappa_{2}^{*^{2}}+\kappa_{3}^{* 2}\right)\right]\right\}
\end{aligned}
$$

8 
In order to integrate over wave number space, spherical coordinates are introduced as follows:

$$
\begin{gathered}
\kappa_{1}=\kappa \cos \theta \\
\kappa_{2}=\kappa \cos \varphi \sin \theta \\
\kappa_{3}=\kappa \sin \varphi \sin \theta
\end{gathered}
$$

For $\overrightarrow{\mathbf{r}}=0$, equations (9) and (10) then become

$$
\overline{u_{i} u_{j}}=\int_{0}^{\infty} \psi_{i j} d \kappa
$$

and

$$
\overline{\tau u_{j}}=\int_{0}^{\infty} \Gamma_{j} d \kappa
$$

where

$$
\psi_{\mathrm{ij}}=\int_{0}^{\pi} \int_{0}^{2 \pi} \varphi_{\mathrm{ij}} \kappa^{2} \sin \theta \mathrm{d} \varphi \mathrm{d} \theta
$$

and

$$
\Gamma_{\mathrm{j}}=\int_{0}^{\pi} \int_{0}^{2 \pi} \gamma_{\mathrm{j}} \kappa^{2} \sin \theta \mathrm{d} \varphi \mathrm{d} \theta
$$

The quantities $\psi_{i j}$ and $\Gamma_{j}$ are functions only of the magnitude of the wave number and represent spectrum functions that have been integrated over all directions in wave number space.

The expressions for the velocity variances $\overline{u_{1}^{2}}, \overline{u_{i} u_{i}}, \overline{u_{2}^{2}}$, and $\overline{u_{3}^{2}}$ can be integrated as follows: 


$$
\begin{aligned}
& \bar{u}_{1}^{2}=\frac{1}{12 \sqrt{\pi} c^{3}(c+1)^{1 / 2}}\left[\frac{3}{2(c+1)^{2}}+\frac{c\left(c^{3}-1\right)}{(c+1)\left(c^{2}+c-2\right)}+\frac{5}{2} \frac{c^{2}\left(c^{3}-1\right)^{2}}{\left(c^{2}+c-2\right)^{2}}\right. \\
& \left.+\frac{3}{4} \frac{\left(c^{2}+c-2\right)}{c+1}+\frac{1}{4} \frac{c}{c+1}\left(2 c^{3}+c^{2}+c-5\right)-\frac{3}{2} \frac{c^{2}\left(c^{3}-1\right)\left(c^{3}-3\right)}{\left(c^{2}+c-2\right)^{2}}\right] \\
& +\frac{1}{6 \sqrt{\pi} c^{3}}\left\{\frac{3}{4} \frac{c^{2}\left(c^{3}-1\right)\left(c^{3}-3\right)}{\left(c^{2}+c-2\right)^{2}} H-\frac{15}{4} \frac{c^{2}\left(c^{3}-1\right)^{2}}{\left(c^{2}+c-2\right)}\left[\frac{c(c+1)^{1 / 2}}{2}-H\right]\right\} \\
& {\overline{u_{i} u_{i}}}^{*}=\frac{1}{6 \sqrt{\pi} c^{3}(c+1)^{1 / 2}}\left[-\frac{c\left(2 c^{6}-c^{3}-1\right)}{4(c+1)\left(c^{2}+c-2\right)}-\frac{3}{4} \frac{c^{2}\left(c^{3}-1\right)^{2}}{\left(c^{2}+c-2\right)^{2}}\right. \\
& \left.+\frac{c\left(c^{3}+1\right)\left(c^{2}+c+1\right)}{8(c+1)}+\frac{c^{2}\left(c^{3}-1\right)\left(c^{3}+2\right)}{8\left(c^{2}+c-2\right)}+\frac{3}{4} \frac{c^{2}\left(c^{3}-1\right)^{2}(c+1)^{1 / 2}}{\left(c^{2}+c-2\right)^{2}} \mathrm{H}\right] \\
& {\overline{u_{2}^{2}}}^{*}={\overline{u_{3}^{2}}}^{*}=\frac{1}{2}\left(\overline{u_{i} u_{i}^{*}}-\overline{u_{1}^{2}}\right)
\end{aligned}
$$

where

$$
\begin{gathered}
\overline{u_{i} u_{j}} *=\frac{\left(x-x_{0}\right)^{5 / 2} \nu / 2}{J_{0} U_{0}^{5 / 2}} \overline{u_{i} u_{j}} \\
H=\left(\frac{c}{c^{2}+c-2}\right)^{1 / 2} \ln \left\{\left(\frac{c^{2}+c-2}{2}\right)^{1 / 2}+\left[\frac{c(c+1)}{2}\right]^{1 / 2}\right\} \quad \text { for } c>1 \\
H=\left(\frac{-c}{c^{2}+c-2}\right)^{1 / 2} \sin ^{-1}\left(\frac{c^{2}+c-2}{-2}\right)^{1 / 2} \text { for } c<1
\end{gathered}
$$


and $\mathrm{c}=\mathrm{U} / \mathrm{U}_{0}$.

Another quantity of importance is the turbulent vorticity tensor $\overline{\omega_{i} \omega_{j}}$. The vorticity spectrum tensor is given in reference 7 as

$$
\Omega_{\mathrm{ij}}=\left(\delta_{\mathrm{ij}} \kappa^{2}-\kappa_{\mathrm{i}} \kappa_{\mathrm{j}}\right) \varphi_{l l}-\kappa^{2} \varphi_{\mathrm{ij}}
$$

As was the case for $\varphi_{i j}$, equation (43) can be integrated over all directions in wave number space to give

$$
\Lambda_{\mathrm{ij}}=\int_{0}^{\pi} \int_{0}^{2 \pi} \Omega_{\mathrm{ij}} \kappa^{2} \sin \theta \mathrm{d} \varphi \mathrm{d} \theta
$$

The vorticity tensor is then given by

$$
\overline{\omega_{i} \omega_{j}}=\int_{0}^{\infty} \Lambda_{i j} d \kappa
$$

Calculated turbulent velocities, vorticities, temperature-velocity correlations, and spectra will be considered in the next section.

\section{RESULTS AND DISCUSSION}

\section{Velocity and Vorticity Variances}

Figure 2 shows turbulent velocity variances $\overline{u_{1}^{2}}, \overline{u_{2}^{2}}$, and $\overline{u_{3}^{2}}$ plotted logarithmically in dimensionless form. Included in the plot is the curve obtained by solving equation (21) as though the effects of strain were absent by omitting the second to sixth terms. This solution gives

$$
\left|\frac{\nu}{\mathrm{s}}\right|^{5 / 2} \frac{\overline{\mathrm{u}_{1}^{2}}}{\mathrm{~J}_{0}}=\frac{\ln ^{-5 / 2} \mathrm{c}}{48 \sqrt{2 \pi}}
$$

For an accelerating flow with uniform strain, the longitudinal component $\overline{u_{1}^{2}}$ decreases more rapidly and the lateral components decrease less rapidly than they would if the effects of strain were absent. For large values of $\mathrm{U} / \mathrm{U}_{0}$, the lateral components reach a steady-state value as observed in Pearson's results (ref. 3). This result differs 


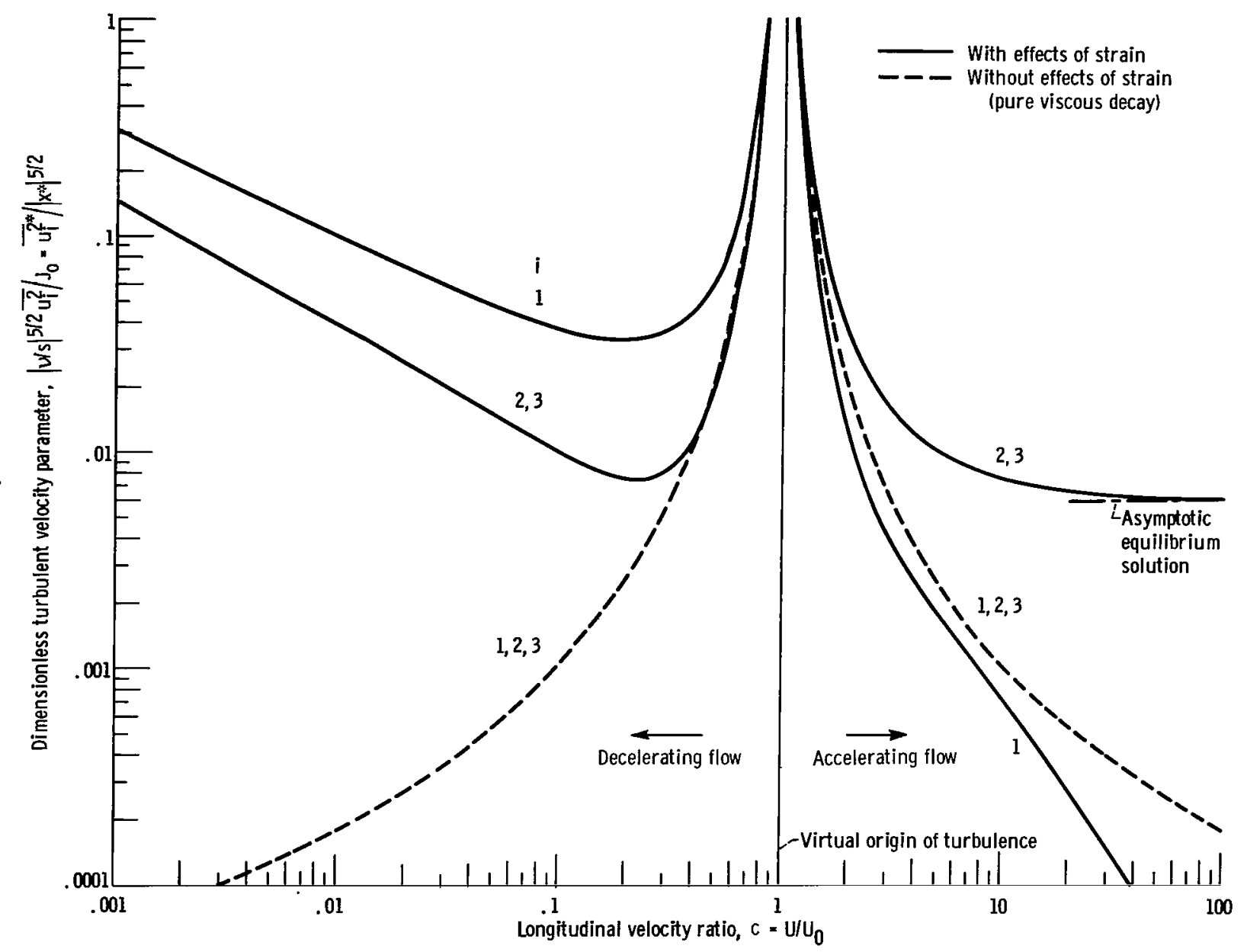

Figure 2. - Dimensionless variance of turbulent velocity components for uniform incompressible strain.

from flow through a converging cone (ref. 2) where the increasing strain rate with distance caused the lateral components to increase without limit as the apex of the cone was approached. The asymptotic equilibrium solution shown in figure 3 is given by

$$
\overline{u_{2}^{2}}=\overline{u_{3}^{2}}=\frac{J_{0}}{96 \sqrt{\pi}}\left(\frac{s}{\nu}\right)^{5 / 2}
$$

Thus, the solution represents a case in which the energy fed into the lateral components by straining action balances the energy dissipated by viscous forces.

For decelerating flow near the virtual origin, both the longitudinal and the lateral components of the velocity fluctuations decrease in the direction of flow. For lower values of $\mathrm{U} / \mathrm{U}_{0}$, all components begin to increase as the effect of normal strain becomes greater than the effect of viscous dissipation. The region of increasing turbulent intensity in the decelerating case was not observed for a cone (ref. 2) where the strain rate $s$ de- 


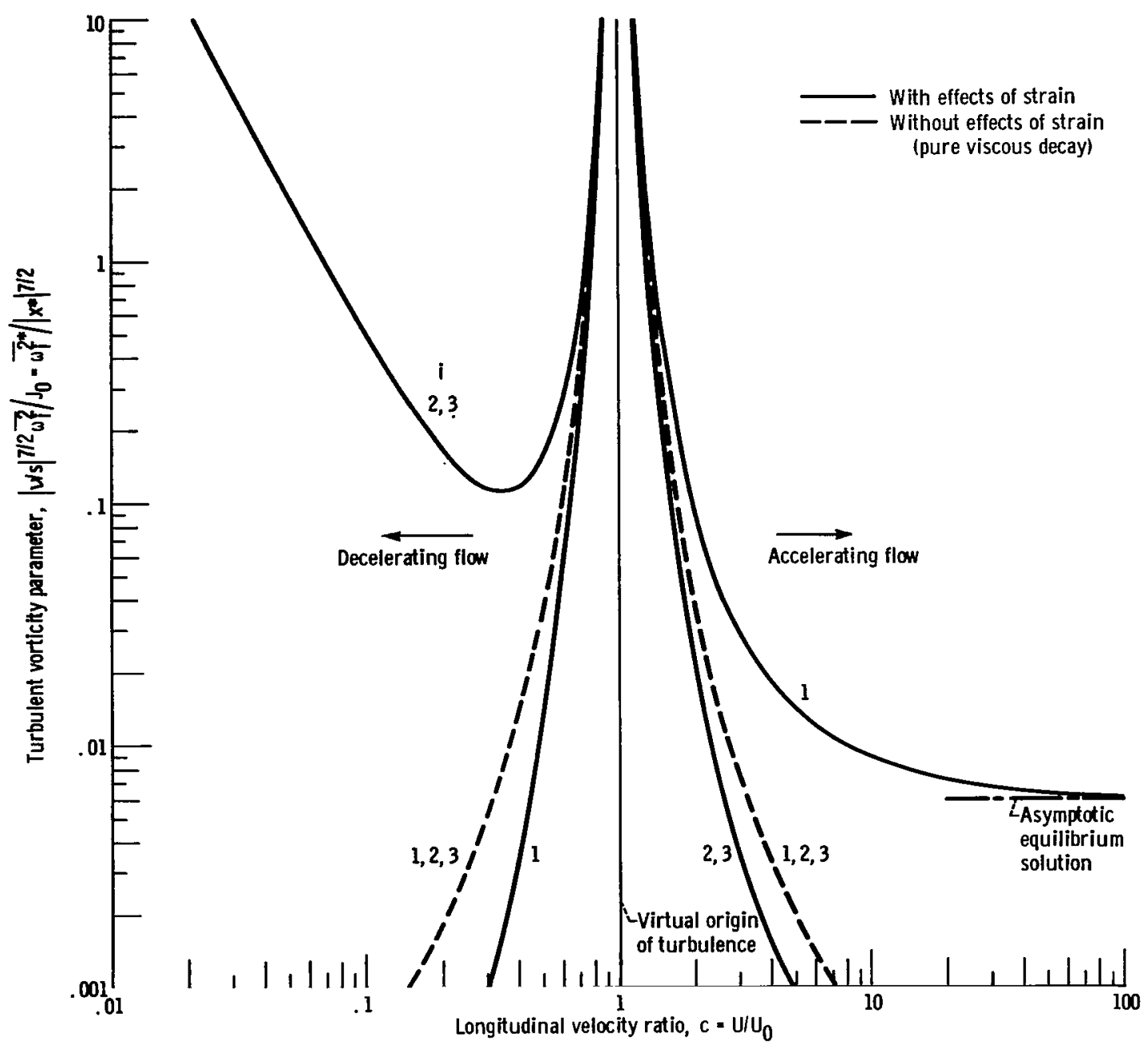

Figure 3. - Dimensionless variance of turbulent vorticity components for uniform incompressible strain.

creased sufficiently with distance to allow the turbulence to decay. As U approaches zero in the present case, the turbulence components will tend to increase without limit. The assumption of local homogeniety will, however, tend to break down in that region, and the turbulence components will remain finite in a real situation. An increase in turbulent fluctuation in the decelerating flow near a stagnation point (in comparison with the free-stream fluctuation) has been observed experimentally in reference 8.

The reasons for the trends observed in the turbulent velocity variances will become clearer if the vorticity variances $\overline{\omega_{i}^{2}}$ plotted in figure 3 are considered. The dashed curves for no effects of strain were obtained from the equation

$$
\left|\frac{\nu}{\mathrm{s}}\right|^{7 / 2} \frac{\overline{\omega_{1}^{2}}}{\mathrm{~J}_{0}}=\frac{5}{192 \sqrt{2 \pi}} \ln ^{-7 / 2} \mathrm{c}
$$


Consider first the accelerating case. Here the trends are opposite to those for the velocity variances in figure 2; that is, the longitudinal vorticity component decays less rapidly, and the lateral components decay more rapidly, than they would if the effects of strain were absent. Thus, the turbulent vorticity tends to become alined in the flow direction. That alinement occurs first, because the longitudinal vortex filaments are strengthened by the stretching action of the mean flow, whereas the lateral filaments are shortened and thus weakened; and, second, because the mean strain rotates the vortex filaments which were originally oblique so that their axes tend to lie in the flow direction. The velocities associated with the turbulent vortex filaments will then tend to lie in the transverse directions, in agreement with the curves for velocity variances in figure 2. As for the lateral components of the velocity variance, the longitudinal component of the vorticity variance approaches an equilibrium solution for large values of $U / U_{0}$ in which the vorticity generated by the mean strain balances that dissipated by viscous action. This solution is given by

$$
\overline{\omega_{1}^{2}}=\frac{\mathrm{J}_{0}}{96 \sqrt{\pi}}\left(\frac{\mathrm{s}}{\nu}\right)^{7 / 2}
$$

For decelerating flow at low values of $\mathrm{U} / \mathrm{U}_{0}$, the lateral components of the vorticity tend to increase, whereas the longitudinal component decreases more rapidly than it would if the effects of strain were absent. Thus, the vortex filaments tend to be alined in the transverse directions. This alinement occurs because the lateral vortex filaments are strengthened by stretching, while the longitudinal components are weakened since they are shortened, and because the axes of vortex filaments which were originally oblique are rotated toward the transverse directions by the stretching action of the mean strain in the transverse directions. With the turbulent vortex filaments mostly alined in the transverse directions, the velocities associated with them can be either in the longitudinal or the transverse directions. This explains why, for low values of $U / U_{0}$, the curves for both the longitudinal and the transverse components of the turbulent velocity variance in figure 2 increase in the flow direction, whereas in the curves for vorticity variance, only the lateral component can increase. The lateral stretching of the vortex filaments intensifies both the longitudinal and the transverse velocity fluctuations.

Relative intensity ratios for turbulence components corrected to eliminate viscous decay are plotted in figure 4. For obtaining the ordinates in this figure, values of turbulent velocity variance with the effects of strain included (solid curves in fig. 2) are corrected to eliminate the effects of decay by dividing them by corresponding values for pure viscous decay (dashed curves in fig. 2). The result (after taking the square root) is divided by $\mathrm{U} / \mathrm{U}_{0}$ to give intensity ratios that are relative to the local mean velocity. In 


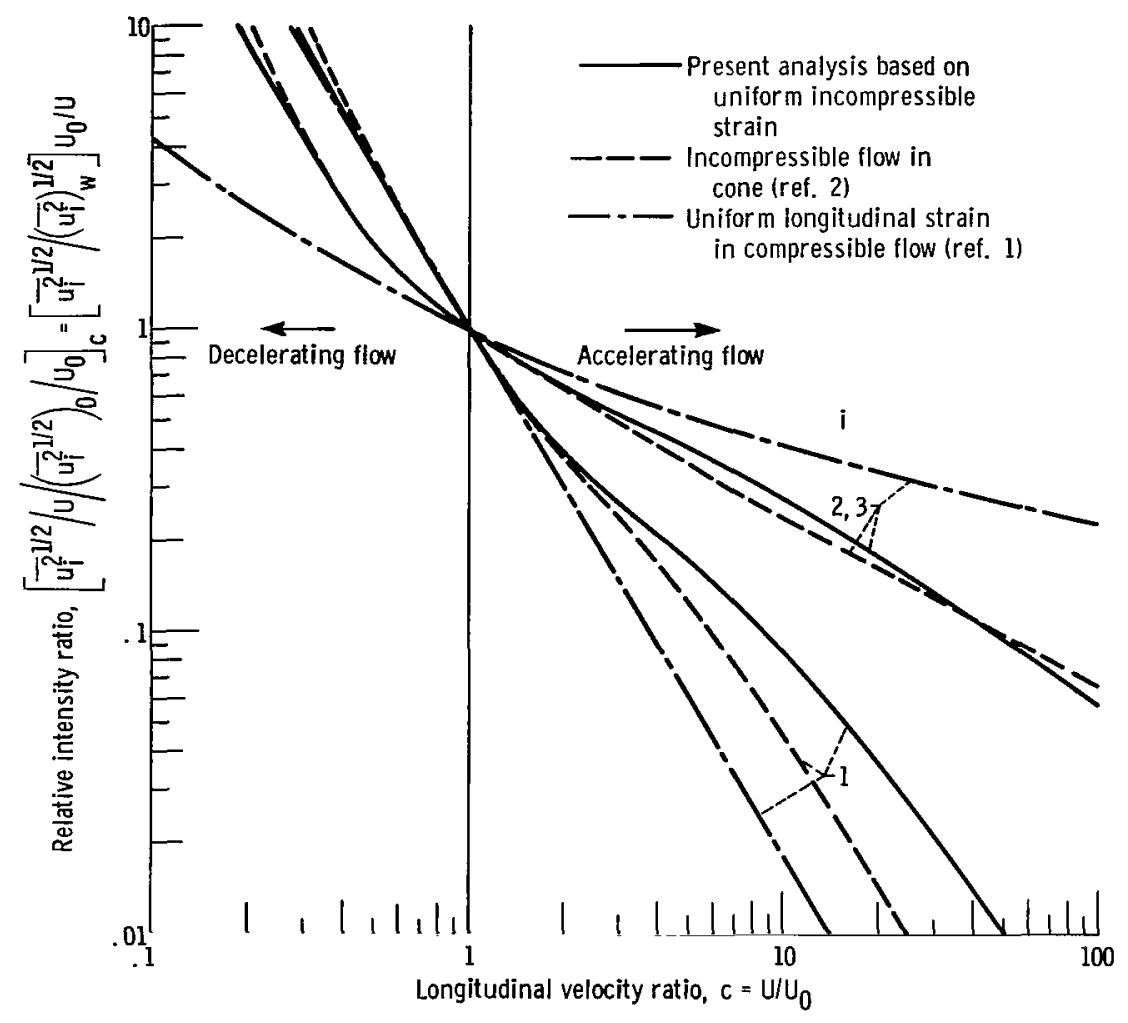

Figure 4. - Comparison of relative intensity ratios for turbulent components corrected to eliminate decay for several situations.

addition to the present results for uniform strain in an incompressible flow, results for flow through a cone (ref. 2) and for uniform longitudinal strain in a compressible flow (ref. 1) are shown in the figure for comparison. The curves for uniform longitudinal strain in a compressible flow were obtained from equations (31) and (32) in reference 1 by noting that $\mathrm{U}_{\mathrm{g}}=\mathrm{U}_{0}-\mathrm{sx}_{0}$ and $\mathrm{U}_{\mathrm{g}} / \nu=\mathrm{U}_{0} / \nu_{0}$. The values for pure viscous decay were obtained by solving equation (23) in reference 1 with all but the first and last terms deleted, and again using $\mathrm{U}_{\mathrm{g}} / \nu=\mathrm{U}_{0} / \nu_{0}$ and $\mathrm{U}_{\mathrm{g}}=\mathrm{U}_{0}-\mathrm{sx}_{0}$. This solution gives $\left(\mathrm{U} / \mathrm{U}_{0}\right)^{-7 / 4}$ for the ordinate of the dot-dashed curve for $i=1$ and $\left(1+U / U_{0}\right)^{1 / 2}\left(U / U_{0}\right)^{-3 / 4} / \sqrt{2}$ for the ordinate of the dot-dashed curve for $i=2,3$.

The curves for the lateral components $(i=2,3)$ for accelerating flow are of particular interest because, as shown in reference 2 , the ordinates of those curves give approximately, for certain conditions, the Stanton number ratio $\mathrm{St} / \mathrm{St}_{0}$ for the heat transfer between the fluid and a wall. In obtaining that relation, the normal strain is assumed to be so large that changes in the Stanton number along the flow are governed by normal strain rather than by shear.

The curves for the lateral components (and thus for the Stanton number ratio) for accelerating flow in figure 4 indicate but a slight difference in the results for uniform 
incompressible strain and for flow in a cone. That is, when plotted in this way, the results at a given $\mathrm{U} / \mathrm{U}_{0}$ for accelerating flow seem to depend but slightly on how the strain $\mathrm{dU} / \mathrm{dx}$ varies along the flow. On the other hand the results for uniform longitudinal strain in a compressible flow lie significantly above the others. These results can evidently be explained by the fact that the stretching of the vortex filaments is more intense in that case, since the lateral compressive strain is absent because of the lack of a radial flow. The dot-dashed curve might be related to heat transfer in a highly heated constant-area tube with fluid density changes along the length, whereas the other two curves are more closely related to nozzle heat transfer in which the effects of compressibility are small (ref. 2, fig. 8).

Figure 5 shows the effect of uniform normal strain on dimensionless longitudinal turbulent heat transfer $\overline{\tau u_{1}}{ }^{*}$. Since $\overline{\tau u_{1}}$ is divided by the temperature gradient $b$, the ordinates can be considered as representing the variation of longitudinal eddy conductivity with $\mathrm{U} / \mathrm{U}_{0}$. Results are given for Prandtl numbers of 0.01 (liquid metals), 1 and 0.7

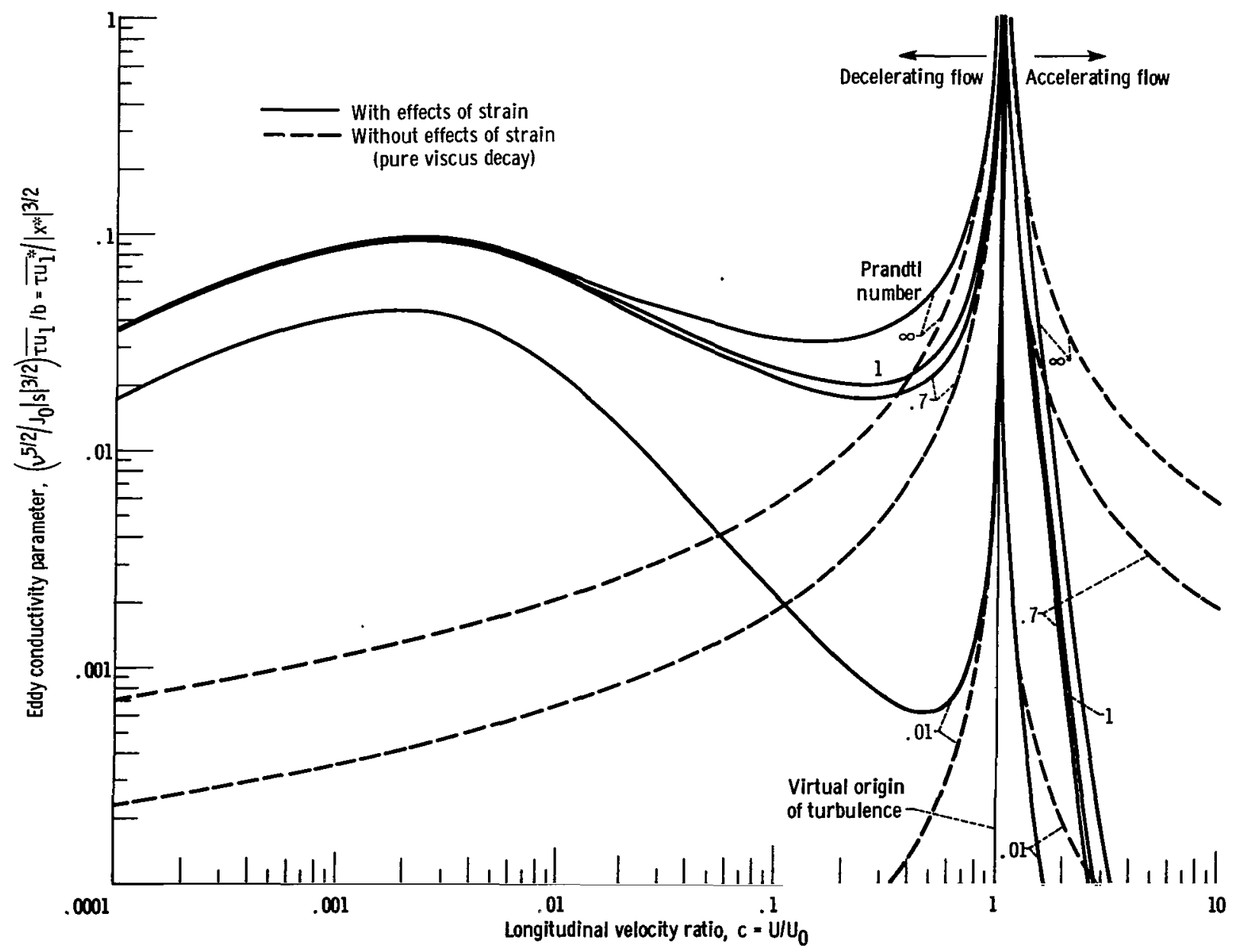

Figure 5. - Dimensionless longitudinal eddy conductivity for uniform incompressible strain. 
(gases), and $\infty$. As Prandtl number decreases, the eddy conductivity decreases, apparently because a turbulent eddy in a high conductivity fluid, such as a liquid metal ( $\mathrm{Pr} \sim 0.01)$ gains or loses heat by conduction as it moves longitudinally and thus transfers heat with relatively low effectiveness (ref. 4, fig. 7). At a given Prandtl number, the trends in the curves are generally similar to those observed for the longitudinal component of the velocity variance $\overline{u_{1}^{2}}$ in figure 2. A notable exception, however, is that $\overline{\tau u_{1}}$ reaches a maximum at low values of $U$ and then approaches zero at $U=0$, whereas $\overline{u_{1}^{2}}$ became indefinitely large as $U$ approached zero. The ratio of maximum to minimum $\overline{\tau u_{1}}$ is greater at low Prandtl numbers.

The curves for decelerating flow in figure 5 illustrate the large increase that normal strain can produce in the longitudinal heat transfer between a body and a stream in the vicinity of a stagnation point when free-stream turbulence is present. The eddy conductivity would decay to very low values (dashed curves) if the effects of strain were absent. (The time available for decay is quite large, since the fluid velocity becomes very small as the stagnation point is approached.) This increase in heat transfer is in agreement with the experiments in reference 9 and the analysis of reference 10 . The increase is evidently produced by the lateral stretching of vortex filaments as assumed in reference 10. Reference 10, however, considered only transverse vortexes, whereas the present analysis considers random vorticity in all directions. The present analysis does not, however, consider the damping effect of the wall at the stagnation point (effect of viscous diffusion), so that the increase in turbulent heat transfer due to normal strain is probably exaggerated here. In fact, an attempt to calculate the heat transfer near a stagnation point by assuming that the maximum in the curve in figure 5 ( $\mathrm{Fr}=0.7$ for gases) corresponds to the maximum eddy conductivity in the boundary layer, and that the minimum in the curve for $i=1$ in figure 2 corresponds to the turbulence level in the undisturbed stream, gave (by dividing one ordinate by the other) increases in total heat transfer considerably higher than those observed experimentally. The results do indicate, however, that the combination of free-stream turbulence and normal strain (or lateral vortex stretching) can be an important factor in increasing the heat transfer in the vicinity of a stagnation point.

\section{Turbulence Spectra}

Dimensionless spectra of components of the velocity and vorticity variances are plotted in figures 6 to 8 . The spectra show how contributions to the dimensionless mean velocity or vorticity fluctuations are distributed among dimensionless wave numbers. Plotted in this way, the curves for no strain $\left(U / U_{0}=1\right)$ reduce to a single curve that does 


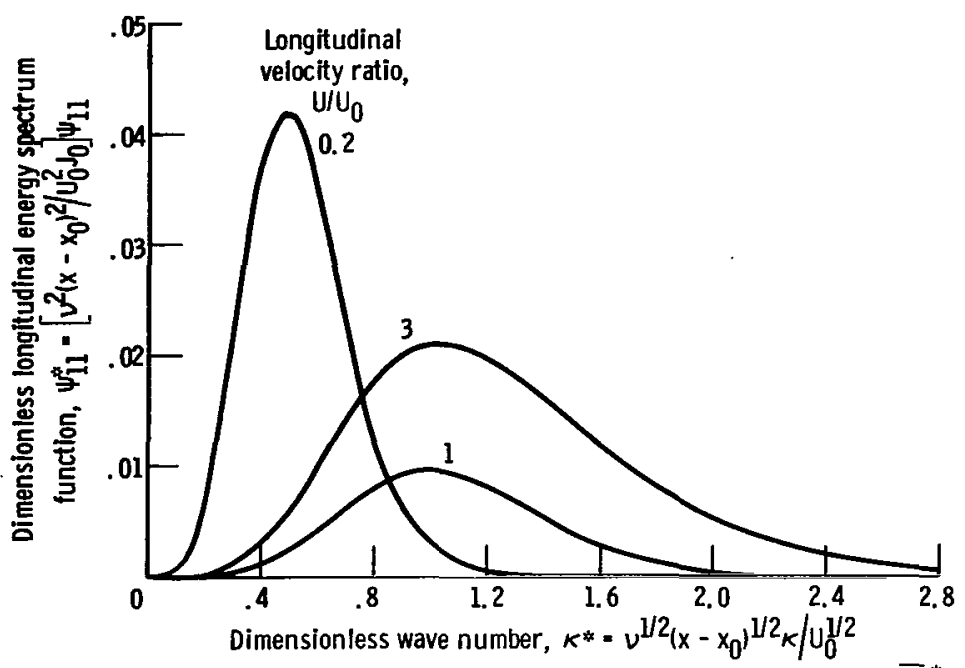

Figure 6. - Spectra of dimensionless longitudinal velocity variance $\bar{u}_{1}^{*}$.

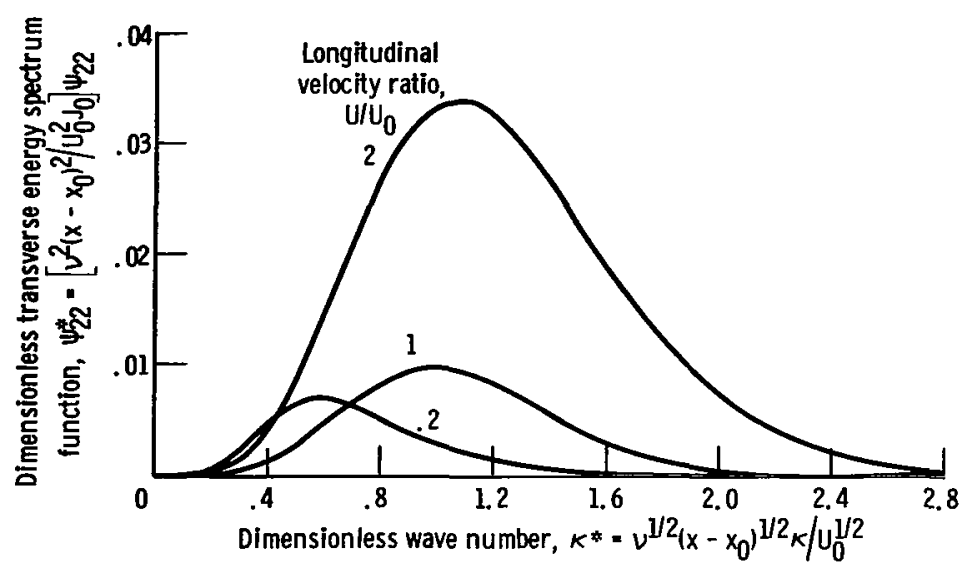

Figure 7. - Spectra of dimensionless transverse velocity variance $\overline{u_{2}^{* *}}$.

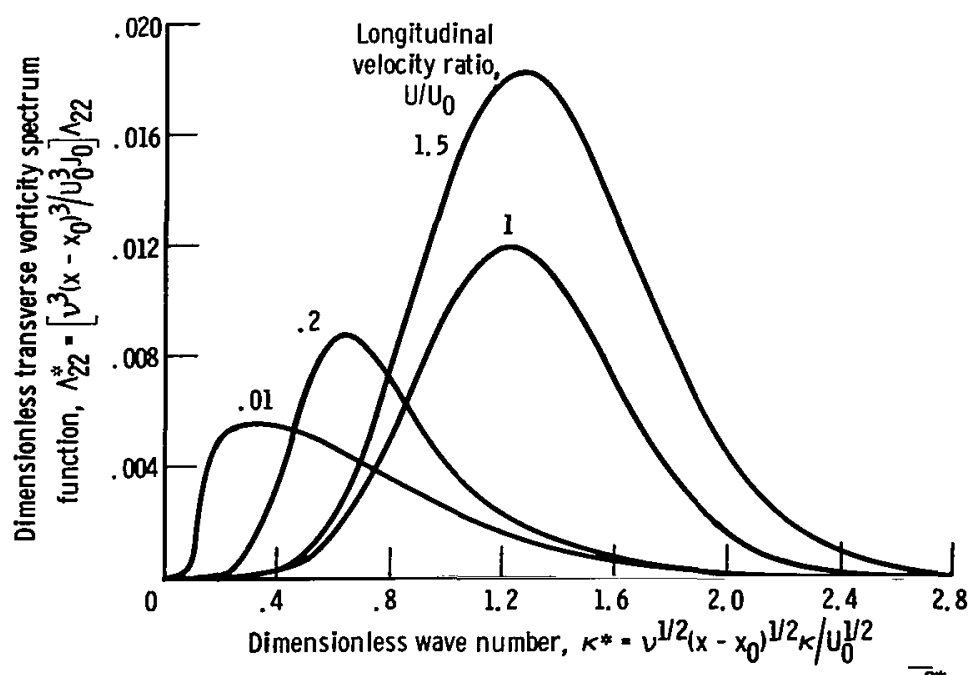

Figure 8. - Spectra of dimensionless transverse vorticity variance $\overline{w_{2}^{2 *}}$. 


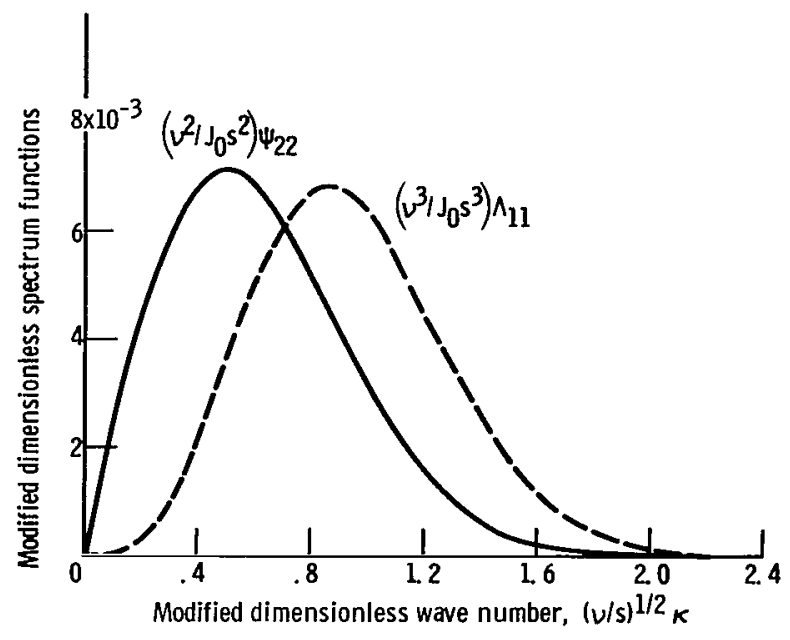

Figure 9. - Asymptotic equilibrium spectra for longitudinal velocity ratio $\mathrm{U} / \mathrm{U}_{0} \rightarrow \infty$.

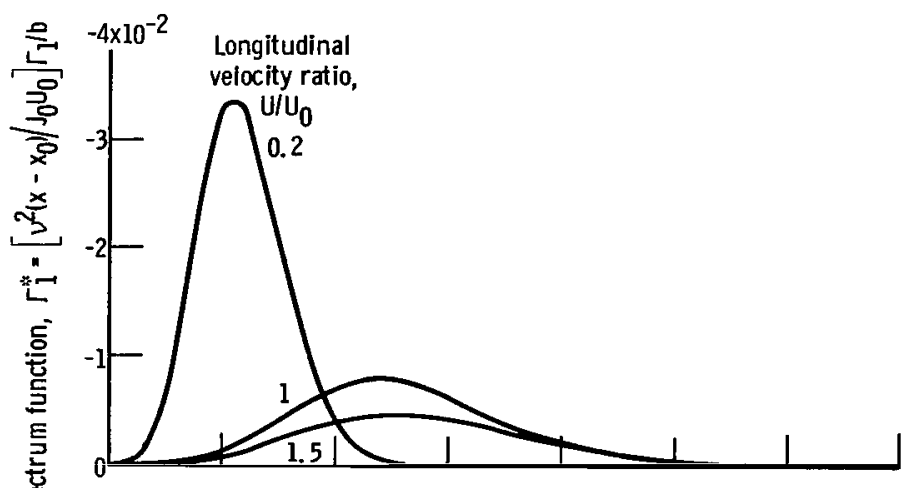

(a) Prandtl number, 0.7 .

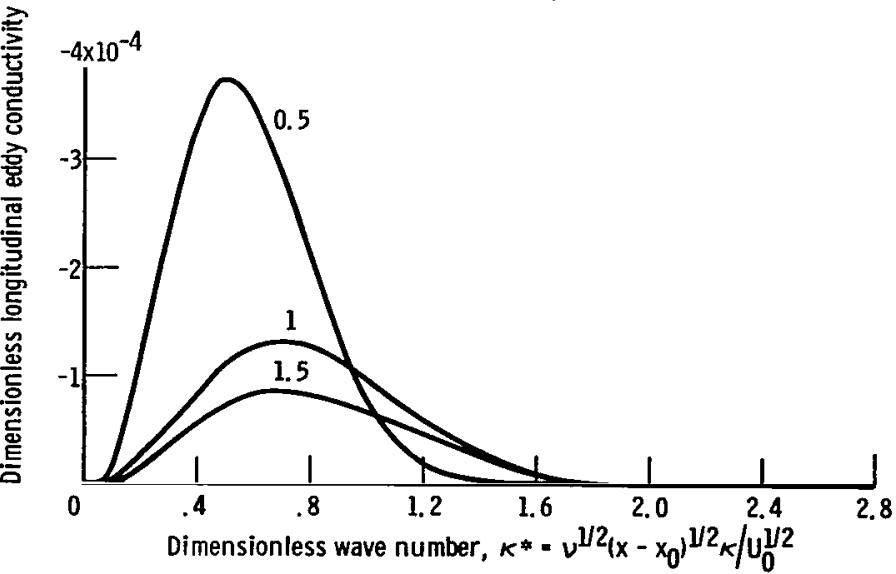

(b) Prandtl number, 0.01 .

Figure 10. - Spectra of dimensionless longitudinal eddy conductivity $\overline{\mathrm{\tau U}}_{1}{ }^{*}$. not change with $x$. Thus, comparison of the curves for various values of $\mathrm{U} / \mathrm{U}_{0}$ shows how strain affects the spectrum at a given position in comparison with the spectrum at the same position with no strain. For instance, figure 8 shows that for decelerating flow $\left(\mathrm{U} / \mathrm{U}_{0}<1\right)$ contributions to the transverse vorticity occur at smaller wave numbers (larger vortexes) than they would for no acceleration. This trend seems to be congruous with the observation in the analysis of reference 10 that only the larger transverse vortexes are amplified by stretching in the neighborhood of a stagnation point. Figures 6 and 7 show that contributions to components of the velocity variance also move to lower dimensionless wave numbers as velocity ratio decreases. For $\mathrm{U} / \mathrm{U}_{0}>1$, the trends are opposite to those for decelerating flow, or contributions to the mean fluctuations move to higher dimensionless wave numbers.

Figure 9 shows spectra corresponding to the asymptotic equilibrium solutions given by equations (47) and (49) for $U / U_{0} \rightarrow \infty$. Note that the dimensionless quantities used in these spectra have been changed from those used in the preceding spectra in order to obtain finite dimensionless quantities for $U \rightarrow \infty$. The spectra in figure 9 are of interest because they show how contributions to the velocity and vorticity variances are distributed among wave numbers for a case in 
which the energy fed into the turbulence by the mean strain exactly balances that dissipated by viscous action. Although the curves are in equilibrium at each wave number, there is not necessarily an equilibrium between production and dissipation at each wave number because energy can be transferred between wave numbers by the stretching of the vortex filaments by the mean velocity gradients as discussed in references $1,2,4$, and 5.

Spectra of the dimensionless longitudinal eddy conductivity $\overline{\tau u_{1}} *$ are plotted in figure $\mathbf{1 0}$ for Prandtl numbers of 0.7 and 0.01 . The shifting of the curves to lower dimensionless wave numbers as $\mathrm{U} / \mathrm{U}_{0}$ decreases is similar to that for the spectra of the longitudinal velocity fluctuations shown in figure 6 .

\section{SUMMARY OF RESULTS}

For an incompressible accelerating flow with uniform strain, the longitudinal velocity fluctuations decreased more rapidly, and the lateral fluctuations decreased less rapidly in the flow direction than they would if the effects of normal strain were absent. For large values of velocity ratio, the lateral components were found to reach a steadystate equilibrium value, as observed in Pearson's results. This result differs from flow through a converging cone, where the increasing strain rate with distance caused the lateral components to increase without limit as the apex of the cone was approached.

For decelerating flow at low values of velocity ratio both the longitudinal and transverse velocity fluctuations increased in the flow direction as the effect of normal strain becomes greater than the effect of viscous dissipation. A somewhat similar increase in velocity fluctuation in the decelerating flow near a stagnation point has been observed experimentally. This region of increasing turbulent intensity in the decelerating case was not observed in an analysis for flow through a diverging cone, where the strain rate decreased sufficiently with distance to allow the turbulence to decay.

Many of the trends observed for the velocity fluctuation components could be explained by the analytical result that the vorticity became alined in the longitudinal direction for accelerating flow and in the transverse directions for decelerating flow.

When the results for turbulent intensity were corrected for viscous decay and divided by local mean velocity, the transverse component for accelerating flow, which can be related to heat transfer between the fluid and a wall, was approximately the same for flow in a cone and for uniform incompressible strain. On the other hand the curve for uniform longitudinal compressible strain lay appreciably above the others, apparently because of the more intense vortex stretching for that case.

The results for longitudinal eddy conductivity in a decelerating flow showed that normal strain could increase that quantity to values considerably above those which it 
would have if the effects of strain were absent. Thus, free-stream turbulence with normal strain (or lateral vortex stretching) could be an important factor in increasing the heat transfer in the vicinity of a stagnation point.

Turbulent vorticity spectra showed that the turbulent vortexes in a decelerating flow tended to be larger at a given location than they would be for no deceleration. The eddies associated with the energy and with the longitudinal eddy conductivity also became comparatively larger. Spectra were also obtained for the energy and the vorticity for the asymptotic equilibrium solutions at large velocity ratios.

\section{Lewis Research Center,}

National Aeronautics and Space Administration,

Cleveland, Ohio, September 16, 1966, 129-01-09-07-22. 


\section{APPENDIX - SYMBOLS}

b

c

$\mathrm{c}_{\mathrm{p}}$

$\mathrm{H}$

h

$\mathrm{J}_{0}$

Pr

$\mathrm{p}$

$\overrightarrow{\mathbf{r}}$

St

s, s (1)

${ }^{s}(2),{ }^{s}(3)$

$\mathrm{T}, \mathrm{T}_{\mathrm{m}}$

t

$\mathrm{U}_{\mathrm{i}},\left(\mathrm{U}_{\mathrm{i}}\right)_{\mathrm{m}}$

$\mathrm{U}_{1},\left(\mathrm{U}_{1}\right)_{\mathrm{m}}, \mathrm{U}$

$u_{i}$

$\overline{u_{i} u_{j}} *$

$\mathrm{x}_{\mathrm{i}},\left(\mathrm{x}_{\mathrm{i}}\right)_{\mathrm{m}}$

$\mathrm{x},\left(\mathrm{x}_{1}\right)_{\mathrm{m}}$

$\mathrm{x}^{*}$

$\alpha$

$\Gamma_{\mathbf{j}}$ longitudinal temperature gradient, $\mathrm{dT} / \mathrm{dx}$

longitudinal velocity ratio, $\mathrm{U} / \mathrm{U}_{0}$

specific heat at constant pressure

defined by eq. (42)

heat-transfer coefficient

constant that depends on initial conditions

Prandtl number, $\nu / \alpha$

pressure

defined in fig. 1

Stanton number, $\mathrm{h} / \rho \mathrm{c}_{\mathrm{p}} \mathrm{U}$

longitudinal velocity gradient, $\partial \mathrm{U}_{1} / \partial \mathrm{x}_{1}=\partial \mathrm{U} / \partial \mathrm{x}=\partial\left(\mathrm{U}_{1}\right)_{\mathrm{m}} / \partial\left(\mathrm{x}_{1}\right)_{\mathrm{m}}$

transverse velocity gradients, $\partial \mathrm{U}_{2} / \partial \mathrm{x}_{2}=\partial\left(\mathrm{U}_{2}\right)_{\mathrm{m}} / \partial\left(\mathrm{x}_{2}\right)_{\mathrm{m}}$,

$$
\partial \mathrm{U}_{3} / \partial \mathrm{x}_{3}=\partial\left(\mathrm{U}_{3}\right)_{\mathrm{m}} / \partial\left(\mathrm{x}_{3}\right)_{\mathrm{m}}
$$

mean temperature

time

mean velocity component

mean longitudinal velocity component

fluctuating velocity component

defined by eq. (41), dimensionless velocity correlation

defined in fig. 1

longitudinal coordinate

$\frac{s\left(x-x_{0}\right)}{U_{0}}$

thermal diffusivity

defined by eq. (37) 
$\Gamma_{1}^{*} \quad$ dimensionless spectrum function for longitudinal eddy conductivity, $\frac{\nu^{2}\left(\mathrm{x}-\mathrm{x}_{0}\right)}{\mathrm{J}_{0} \mathrm{U}_{0}} \frac{\Gamma_{1}}{\mathrm{~b}}$

$\gamma_{\mathbf{i}} \quad$ defined by eq. (10)

$\gamma_{i}^{*} \quad$ defined by eq. (30)

$\delta_{i j} \quad K r o n e c k e r$ delta

$\zeta^{\prime}$ defined by eq. (11)

$\theta, \varphi \quad$ angular coordinates (see eq. (33))

$\kappa_{i} \quad$ wave number component

$\kappa_{i}^{*} \quad$ defined by eq. (28)

$\Lambda_{i j} \quad$ vorticity spectrum function, defined by eq. (44)

$\Lambda_{\mathrm{ij}}^{*} \quad$ dimensionless vorticity spectrum function, $\Lambda_{\mathrm{ij}} \frac{\nu^{3}\left(\mathrm{x}-\mathrm{x}_{0}\right)^{3}}{\mathrm{U}_{0}^{3} \mathrm{~J}_{0}}$

$\nu \quad$ kinematic viscosity

$\rho$ density

$\tau \quad$ temperature fluctuation

$\overline{\tau \mathrm{u}_{1}} \quad$ longitudinal temperature-velocity correlation

$\overline{\tau u}_{1} * \frac{\nu^{5 / 2}\left(\mathrm{x}-\mathrm{x}_{0}\right)^{3 / 2}}{\mathrm{~J}_{0} \mathrm{U}_{0}^{3 / 2}} \frac{\overline{\tau \mathrm{u}_{1}}}{\mathrm{~b}}$

$\varphi_{\mathrm{ij}} \quad$ defined by eq. (9)

$\varphi_{\mathrm{ij}}^{*} \quad$ defined by eq. (29)

$\psi_{i j} \quad$ energy spectrum function, defined by eq. (34)

$\psi_{\mathrm{ij}}^{*} \quad \psi_{\mathrm{ij}} \frac{\nu^{2}\left(\mathrm{x}-\mathrm{x}_{0}\right)^{2}}{\mathrm{U}_{0}^{2} \mathrm{~J}_{0}}$

$\Omega_{i j} \quad$ defined by eq. (43)

$\overline{\omega_{i} \omega_{j}} \quad$ turbulent vorticity variance 


$$
\bar{\omega}_{i} \omega_{j}^{*} \quad \omega_{i} \omega_{j} \frac{\nu^{7 / 2}\left(x-x_{0}\right)^{7 / 2}}{\mathrm{U}_{0}^{7 / 2} \mathrm{~J}_{0}}
$$

Subscripts:

c corrected to eliminate viscous decay

$\mathrm{m}$ at point $\mathrm{P}_{\mathrm{m}}$ (see fig. 1)

w without effects of strain

0 at virtual origin of turbulence where turbulent energy would be infinite (It is assumed that turbulence is isotropic at $x_{0}$ and that strain begins to act there.)

1 in longitudinal or flow direction

2,3 in transverse direction

Superscripts:

,

at point $P^{\prime}$ (see fig. 1)

* on dimensionless quantities

- $\quad$ over averaged quantities 


\section{REFERENCES}

1. Deissler, Robert G. : Effect of Uniform Longitudinal Strain Rate on Weak Homogeneous Turbulence in a Compressible Flow. NASA TN D-2800, 1965.

2. Deissler, Robert G.: Weak Locally Homogeneous Turbulence in Idealized Flow Through a Cone. NASA TN D-3613, 1966.

3. Pearson, J. R. A. : The Effect of Uniform Distortion on Weak Homogeneous Turbulence. J. Fluid Mech., vol. 5, pt. 2, Feb. 1959, pp. 274-288.

4. Deissler, Robert G.: Turbulent Heat Transfer and Temperature Fluctuations in a Field with Uniform Velocity and Temperature Gradients. Int. J. Heat Mass Transfer, vol. 6, no. 4, Apr. 1963, pp. 257-270.

5. Deissler, Robert G. : Effects of Inhomogeneity and of Shear Flow in Weak Turbulent Fields. Phys. Fluids, vol. 4, no. 10, Oct. 1961, pp. 1187-1198.

6. Hildebrand, Francis B.: Advanced Calculus for Engineers. Prentice-Hall, Inc. , 1949 , p. 374 .

7. Batchelor, George K. : The Theory of Homogeneous Turbulence. Cambridge Univ. Press, 1953.

8. Kuethe, A. M.; Willmarth, W. W.; and Crocker, G. H.: Stagnation Point Fluctuations on Bodies of Revolution with Hemispherical Noses. Rep. No. 02753-2-F (AFOSR TR 60-65), Michigan University, College of Eng., June 1960.

9. Kestin, J.; Maeder, P. F.; and Sogin, H. H. : The Influence of Turbulence on the Transfer of Heat to Cylinders near the Stagnation Point. Z. Angew. Math. Phys., vol. 12 , no. 2, 1961, pp. 115-132.

10. Sutera, S. P.: Vorticity Amplification in Stagnation-Point Flow and its Effect on Heat Transfer. J. Fluid Mech., vol. 21, pt. 3, Mar. 1965, pp. 513-534. 
"The aeronautical and space activities of the United States shall be conducted so as to contribute . . . to the expansion of buman knowledge of phenomena in the atmosphere and space. The Administration shall provide for the widest practicable and appropriate dissemination of information concerning its activities and the results tbereof."

-Nafional Aeronautics and SPACE ACt Of 1958

\section{NASA SCIENTTIFIC AND TEĊHNICAL PỦBLICẢTIONS}

TECHNICAL REPORTS: Scientific and technical information considered important, complete, and a lasting contribution to existing knowledge.

TECHNICAL NOTES: Information less broad in scope but nevertheless of importance as a contribution to existing knowledge.

TECHNICAL MEMORANDUMS: Information receiving limited distribution because of preliminary data, security classification, or other reasons.

CONTRACTOR REPORTS: Technical information generated in connection with a NASA contract or grant and released under NASA auspices.

TECHNICAL TRANSLATIONS: Information published in a foreign language considered to merit NASA distribution in English.

TECHNICAL REPRINTS: Information derived from NASA activities and initially published in the form of journal articles.

SPECIAL PUBLICATIONS: Information derived from or of value to NASA activities but not necessarily reporting the results of individual NASA-programmed scientific efforts. Publications include conference proceedings, monographs, data compilations, handbooks, sourcebooks, and special bibliographies.

Details on the availability of these publications may be obtained from:

SCIENTIFIC AND TECHNICAL INFORMATION DIVISION

Washington, D.C. 20546 